\title{
Efficacy of Intraoperative Ultrasound in Resection of Posterior Fossa Lesions
}

\author{
Ahmed Nagaty*, Ahmad Elsabaa, Hisham Anwer \\ Neurosurgery Department, Ain Shams University, Cairo, Egypt \\ Email address: \\ Ahmednagaty@med.asu.edu.eg (A. Nagaty), Ahmed.abdelrhem@med.asu.edu.eg (A. Elsabaa), Hishambando@yahoo.com (H. Anwer) \\ ${ }^{*}$ Corresponding author
}

To cite this article:

Ahmed Nagaty, Ahmad Elsabaa, Hisham Anwer. Efficacy of Intraoperative Ultrasound in Resection of Posterior Fossa Lesions. International Journal of Neurosurgery. Vol. 3, No. 2, 2019, pp. 13-20. doi: 10.11648/j.ijn.20190302.11

Received: September 3, 2019; Accepted: October 8, 2019; Published: October 20, 2019

\begin{abstract}
To evaluate the important role of Intraoperative Ultrasound (IOUS) in measurement of pre \& postoperative resection volume of an intra-axial posterior fossa lesions and to compare these results with that measured in pre and postoperative MRI brain. Also, to assess the principal surgeon opinion regarding the ability of IOUS in localization and differentiating the borders of those lesions, and his comfortability during handling of this device. There are different methods, which are helpful to localize precisely and allow maximum tumor resection, such as neuro-navigation, intraoperative MRI, 5aminolevulinic acid, as well as IOUS. Every method has its pros \& cons. Although Intraoperative MRI Provides real time updated images, however it's not available in most of neurosurgery centers in developing countries. So, with these financial and ergonomic limitations of the Intraoperative MRI, usage of Intraoperative Ultrasound could be considered as a potential competitor in developing world. This is a prospective study conducted at Ain Shams University hospital in the period from February 2016 to July 2018, we enrolled 23 patients presented with posterior fossa intra axial neoplasms that operated with Intra Operative Ultrasound (IOUS) assistance. IOUS was able to well localize the lesions in $96 \%$ of cases and it was able to differentiate solid and cystic parts in all the cases. IOUS was able to define well the lesion borders in 20/23 (87\%) of cases. There was no difference between volumes calculated from preoperative MRI and the pre-resection IOUS, also there was no significant difference between the residual volume detected by the IOUS and the postoperative MRI brain. Principal surgeon reported overall great impression of the IOUS in 18/23 (78\%) of cases. Mean total additional time of usage of the IOUS was $27 \mathrm{~min}$. IOUS was found to be valuable in localization of the lesions, differentiating solid and cystic parts and detection of the residual volume in the posterior fossa intra axial tumors.
\end{abstract}

Keywords: Neuro-navigation, Intra-operative Ultrasound, IOUS, Intraoperative MRI, Posterior Fossa, Intra-axial Lesions

\section{Introduction}

Posterior fossa lesions are considered as critical brain lesions. This can be explained by the limited space of the posterior fossa and high risk of involvement of vital centers. Surgical intervention of such lesions is considered of the top neurosurgical challenges [1].

Several surgical techniques and technological innovations have been recently introduced to help the surgeon achieve the maximal safe resection of the tumor while reducing the odds of post-operative complications. Some examples are represented by the intraoperative use of neurophysiological monitoring and the use of the awake surgery technique.
Moreover, in recent years, new methods, including intraoperative diagnostic imaging (Intra-Operative Magnetic Resonance-IOMR, Intra-Operative Computed TomographyIOCT, Intra-Operative Ultrasound-IOUS) and dyes (fluorescence) have become relevant in neuro-oncological surgery, allowing for a more radical and safer tumor resection [2].

Some of these innovations, such as the IOMR, are expensive and not available in every neurosurgical center. Therefore, in the last few years, great attention has been paid to the possible intraoperative use of ultrasound (IOUS) guidance for brain tumor surgery [3].

In the 1980s, Rubin et al published their first experience with the neurosurgical application of intraoperative 
ultrasound (IOUS). They found that ultrasound is friendly to use and capable of demonstrating normal brain anatomy as well as identifying and localizing brain lesions. Since the 1990s, with the advance in imaging studies, real-time IOUS retained important role in neurosurgery for localization of subcortical lesions in eloquent brain areas [4].

IOUS is not a new technology, but it is accurate in distinguishing tumor from normal parenchyma and allows a real-time intraoperative visualization. IOUS is cheap, easily repeatable, safe for the patient, potentially available in all neurosurgical centers [3].

\section{Patients and Methods}

This is a prospective study, conducted at Ain Shams University hospitals in the period from February 2016 to July 2018. This study enrolled 23 patients that operated using intraoperative ultrasound IOUS assistance. Patients presented with cerebellar, brainstem or fourth ventricular intra-axial lesions were included in this study with no age or sex restrictions. Extra axial posterior fossa lesions e.g.
Cerebellopontine angle lesions were excluded.

The primary aim of our study was to evaluate the efficacy of IOUS in detection of residual volume after resection of intra-axial posterior fossa lesions. While secondary aims were to evaluate efficacy of IOUS in localization of posterior fossa intra-axial lesions and defining its borders, evaluate efficacy of IOUS in detection of pre-resection volume in comparison to preoperative MRI, and to evaluate the main surgeon opinion and comfortability during handling of IOUS device.

Checklist for data collection included:

1. Age and sex.

2. Presentation:
a. Increased intracranial pressure ICP.
b. Cranial nerves deficits.
c. Ataxia: Truncal or limb ataxia.
d. Weakness.
e. Torticollis.
f. Lansky Performance Scale (LPS) in children 100
g. Karnofsky Performance Scale (KPS) in adults.90

Table 1. Karnofsky/Lansky performance scale [5].

\begin{tabular}{lll}
\hline & Karnofsky scale (Adults) & Lansky Scale (children) \\
\cline { 2 - 3 } & Non-dependent & Normal activity \\
\hline 100 & Normal, no complains and no evidence of disease & Fully active \\
90 & Able to carry on normal & Minor restriction in physically strenuous play \\
& Normal activity with effort & Restricted in strenuous play, tires more easily, otherwise active. \\
& Unable to work & Mild to moderate restriction in activity \\
70 & Cares for self, unable to carry on normal or to do active work & Both greater restriction of and less time spent in active play. \\
60 & Requires occasional assistance but is able to care for most needs. & Ambulatory up to 50\% of time, limited active play \\
50 & Requires considerable assistance and frequent medical care & Considerable assistance required for any active play \\
40 & Disabled, requires special care and assistance. & Able to initiate quite activities \\
30 & Severely disabled. & Needs considerable assistance for quite activity \\
20 & Very sick, hospitalization necessary & Limited to very passive activity (e.g. TV) \\
10 & Fatal process progressing rapidly & Completely disabled \\
\hline
\end{tabular}

All patients enrolled in the study were subjected to preoperative contrasted MRI and CT brain to evaluate volume of the lesion in the preoperative MRI: Calculated by the ellipsoid volume formula $(1 / 2 \mathrm{~A} \times \mathrm{B} \times \mathrm{C})$, where $\mathrm{A}$ and $\mathrm{B}$ represent the two axial perpendicular dimensions and $C$ represents the craniocaudal dimension.

\subsection{Operative Data Collection}

Pre-resection volume of the lesion by the IOUS was calculated by the ellipsoid volume formula after measuring 2 dimensions in the axial orientation of the IOUS. The craniocaudal dimension of the lesion was measured in the sagittal orientation of the IOUS. When the IOUS detected a residual of the lesion at the end of resection, the volume of this residual lesion was calculated by the same described method for the pre-resection volume. Additional time for the use of IOUS was calculated from the time the surgeon asked for the IOUS till the end of the scanning and the start of next step of the surgery.

Total additional time of IOUS use is the summation of the additional time required for all the scans done during the surgery.

\subsection{Questionnaire}

The main surgeon impression of the IOUS was assessed by a questionnaire collecting the following data:

Identification of the extent of craniectomy.

i) Localization of the lesion that was defined as the distinction in echogenicity

between the lesion and cerebellum. IOUS localization was classified into 3 categories:

a) Good, if the lesion is well distinct from surrounding brain tissue.

b) Fair, if the lesion is fairly localized from the surrounding brain.

c) Poor, if the lesion cannot be differentiated from surrounding normal brain structure.

ii) Definition of lesions borders: The IOUS defined lesion borders either as: well defined or fairly defined or poorly defined (Figure 1).

a) Well defined: If lesion is defined all around the borders from the normal brain tissue.

b) Fairly defined: If more than $50 \%$ of the borders are defined. 
c) Poorly defined: If less than $50 \%$ of the tumor borders are defined.
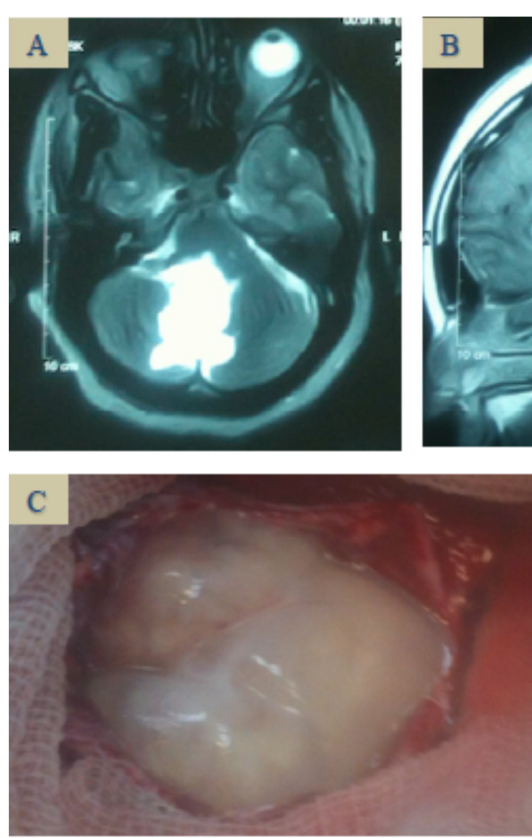
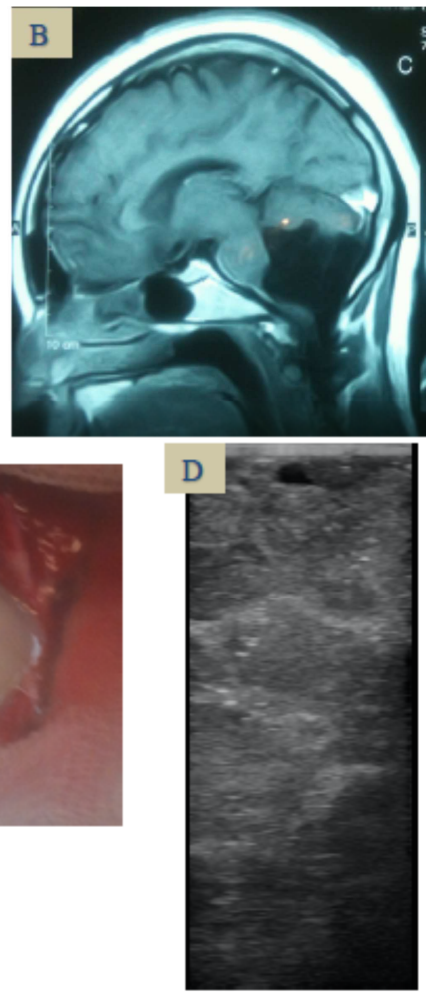

Figure 1. A) Axial T2 MRI of a case of 4th ventricular epidermiod. B) T1 WI of the same case. C) Intraoperative appearance of epidermiod. D) IOUS image of epidermiod case.

ii) Differentiation of the solid from the cystic parts of the lesion.

iii) Any difficulty with the IOUS ergonomics in the operating theatre.

iv) How the surgeon was satisfied with IOUS: this was assisted on a scale from 1-3: "1" is great, I will use it in all my future cases, " 2 " is intermediate, and " 3 " I don't think it is helpful at all.

\subsection{Hardware}

We used the GE logic book XP ultrasound, General electric USA.

At our institute we have 2 probes (Transducers). The first one is the linear intraoperative probe (i12 L) and the second one is the microconvex probe $(8 \mathrm{C})$. When we first introduced the IOUS in our department we used to use the linear intraoperative probe. It has the advantage of having a small footprint about $2.5 \times 1 \mathrm{~cm}$, however it has a narrow field of view and there is no virtual convex feature. This probe doesn't allow us to see borders of the lesion with its narrow field of view. After we encountered some troubles with the linear probe in the cranial surgeries, we introduced the microconvex probe, which allows a wider field of view while having a small footprint. Now, we prefer to use the linear probe in the spinal cord cases and the microconvex probe with the cranial cases.
The IOUS transducer was submersed in dialdehyde (Cidex) for 20 minutes for disinfection. After that, the probe is draped in a sterile sleeve. In some cases, we preferred to put the transducer in sterile gloves with K-Y gel inside the gloves. In other cases, we use the sterile probe directly.

The frequency of the ultrasound was adjusted according to the depth of the lesion. A higher frequency allows visualization of superficial cortical lesions. However, lower frequency allows visualization of deeper lesions. The frequency range in our device is from 6-10. In most cases we used a frequency of $6 \mathrm{MHz}$.

\subsection{Description of the Technique}

The prone position was used in all our cases. This position allows filling the tumor cavity with saline at the end of resection minimizing air between the saline and the IOUS probe, which improves image quality of the IOUS.

The head was fixed on the 3 pins fixator or horseshoe headrest depending on the age of the patient. The head of the table was elevated above the level of the heart of the patient. The neck was flexed that the chin was kept 2-finger breadth away from the chest. Shoulder traction was done when needed. We used the standard suboccipital approach with midline skin incision extending from inion to $\mathrm{C} 1-2$ spinous process. This allowed dissection through avascular plan.

The extent of the craniectomy was modified before dural opening with the aid of the IOUS. So if any of the lesions' borders happen to lie underneath the bone edges of the craniectomy, extension of bone would be done. Cranial, caudal, medial and lateral borders of the lesion were identified with the aid of the IOUS. The IOUS was used to scan starting from normal cerebellar tissue towards the lesion to identify each border.

Measurement of the lesion dimensions was done using the IOUS in 3 dimensions; antero-posterior and medio-lateral on axial orientation of the IOUS and the third dimension on the sagittal orientation of the IOUS.

IOUS was used to differentiate solid from cystic parts of the lesion when the lesion has both a cystic and solid parts. Color flow Doppler was used to determine vascular structures surrounding the lesion. For midline lesions opening of the dura is done in a Y-shaped fashion. Control of the occipital and circular sinus in pediatric age groups would be done with suturing or hemostatic clips.

In lateral cerebellar lesions, the dura is opened in cruciate fashion according to the IOUS data regarding the most surfacing part of the lesion. This step minimizes the exposed cerebellar cortex after opening of the dura. Another IOUS scan was done after opening of the dura to help in determining best surgical corridor to attack the tumor (extent of vermian incision or cerebellar corticotomy). In the case of midline and paramidline tumors we used the standard transvermian or telovelar approaches. In the case of hemispheric tumors, Direct Corticotomy was done from the shortest distance to the lesion or to the solid nodule incase the lesion had both solid and cystic parts. The IOUS was used after that whenever the surgeon needed to provide real time updates. 


\subsection{Residual Detection}

IOUS scanning was done at the end of resection for detection of any residual. The tumor cavity was filled with saline to minimize any air between transducer and the lesion. Also, we measured the dimension of any residual detected. At the end of resection, the volume of any residual lesions identified by the IOUS was calculated. In some cases, the residual lesion was left behind because it is strictly adherent to the brainstem or patient develop vital instability with manipulation of it.

\subsection{Post-operative Evaluation}

MRI brain was done in the first 72 hours of the postoperative period.

If any residual lesion was found in the post-operative MRI, its volume was calculated and compared to that detected by the IOUS.

\subsection{Statistical Analysis}

After data gathering, data were classified into continuous or categorical date. Mean, Standard error of the mean (SEM), Standard deviation (SD), ranges and medians were used in analysis of continuous data collected in the study.
Proportions or percentage were used to present the categorical data collected in the study.

Pre-resection IOUS volumes were compared to preoperative MRI volumes.

Residual lesions volumes were compared with that detected by the IOUS and postoperative scanning. Student $t$ test was used to compare calculated volumes of lesions detected on both IOUS with volumes calculated from preoperative and postoperative scanning. GraphPad online calculator was used for all statistical analysis of the study.

\section{Results}

This study enrolled 12 females and 11 male patients. Mean age of enrolled patients was 22 years old. Thirteen patients were adults and 10 patients were children.

Patients enrolled in this study harbored different pathological diagnosis. Most common pathology in our series was medulloblastoma. We had 6 cases of medulloblastoma.

Ependymoma comes in the second rank with 4 cases in the whole series. Pilocytic astrocytoma and hemangioblastoma were the third most common pathologies in our study. Other pathologies included: Epidermoid, abscess, astrocytoma grade III, metastasis and brainstem glioma.

Table 2. No of different pathologies included in the study.

\begin{tabular}{|c|c|c|c|c|c|}
\hline Total & Medulloblastoma & Ependymoma & Hemangioblastoma & Pilocytic Astrocytoma & Others \\
\hline 23 & 6 & 4 & 3 & 3 & 7 \\
\hline
\end{tabular}

Symptoms of increased intracranial tension were present in all enrolled cases in this study. Second most common presenting symptom was ataxia 22/23 (11 cases with truncal ataxia and 11 with limb ataxia). Lower cranial nerves (Glossopharyngeal IX, accessory XI and hypoglossal XII nerves) affection was found in $6 / 23(26 \%)$ of cases in our series. at the time of presentation, $3 / 23(13 \%)$ of enrolled cases had abducens nerve (CN VI) palsy. Torticollis was the presenting symptom in $1 / 23(4 \%)$ of cases. Weakness was the presenting symptom in $1 / 23(4 \%)$ of cases.

\subsection{Evaluation of the IOUS}

Evaluation of the accuracy of the IOUS in volume detection:

1. The mean preoperative tumor volume calculated by the MRI was $12.87 \mathrm{cc}$, SD 4.61 and SEM 0.96. The mean pre-resection volume calculated from IOUS was 12.96 cc, SD 4.46 and SEM 0.93. The difference in the mean between the two groups was 0.09 with a non-significant $95 \%$ confidence interval ( -2.78 to 2.61$)$. $\mathrm{P}$ value was 0.95 , which is nonsignificant.

This suggests that there was no difference between volumes calculated from preoperative MRI and the preresection IOUS.

1. IOUS was able to detect residual lesions in $13 / 23(57 \%)$ of cases. The postoperative MRI confirmed the presence of the residual lesion in $12 / 13(92 \%)$ of these cases.
2. The mean residual volume calculated from the IOUS is $1.48 \mathrm{cc}$, SD 2.12, SEM 0.44. We compared that to the volumes calculated from the postoperative scanning. The mean residual volume calculated from postoperative scanning was $1.45 \mathrm{cc}$, SD 2.06 and SEM 0.43 . The mean difference between the two calculated volumes was 0.044 with $95 \%$ CI (-1.2 to 1.28$)$. P value was 0.94 denoting no significant difference between the residual volumes detected by the IOUS or the postoperative MRI.

\subsection{Extent of Resection}

We achieved gross total resection in 11/23 (49\%) cases. When we classified the extent of resection into more or less than $85 \%$. It was found that, extent of resection $>85 \%$ was achieved in 19/23 (83\%) of the cases.

\subsection{Analyses of the Main Surgeon Opinion Questionnaire}

i) Identifying the appropriate extent of craniectomy:

The IOUS helped to identify extent of the craniectomy in $13 / 23(56 \%)$ cases. By identifying the extent of craniectomy we mean, that we extend the craniectomy in specific directions depending on location of the lesion in relation to the craniectomy done.

ii) Localization of the lesion:

According to the methodology we stratified the degree of localization into good, fair and poor. Good localization was 
achieved in 22/23 (96\%) of cases. Poor localization was reported in only one case

iii) Differentiating solid and cystic part: In our series we had $9 / 23(40 \%)$ patients with partially cystic lesions. The use of the IOUS helped to differentiate between the cystic and solid parts in all those lesions.

iv) Defining lesion border: IOUS was able to well define lesion borders in 20/23 (87\%) of cases.

Analysis of overall impression by the main surgeon revealed: Principal surgeon reported overall great impression in $18 / 23(78 \%)$ of cases and moderate impression in $5 / 23$ $(22 \%)$ of cases with no reported cases of being not of any help. There was no ergonomics difficulty reported by any of the principal surgeon who used the IOUS.

\subsection{Additional Time of Using the IOUS}

We calculated the total additional time that is required for using IOUS in 10/23 cases and we found that mean total additional time of usage of IOUS in the surgery was 27 minutes in these cases, with a range from 15 minutes to 60 minutes.

\section{Discussion}

Resection of posterior fossa neoplasms needs surgical navigation in order to guide maximum resection safely, especially with the growing evidence of the effective cure with maximizing the extent of brain tumor excision $[6,7]$.

Although the intra-operative MRI is available in few centers worldwide which provides precise real time updates during the resection of brain tumors. But, it has many limitations as: It needs large space in the operation room, requires specific MRI compatible surgical instruments and expensive. All these limitations affect its availability in most of medical centers in developing countries. On the other hand, IOUS is a cheap device which can be provided in most of these centers. From this prospective study; we are presenting our experience of using IOUS in resection of posterior fossa intra axial lesions as a cost-effective tool.

\subsection{Value of the IOUS}

i) Localization of lesions:

Hammoud et al concluded in their study of 70 patients with intra-axial brain lesions that IOUS was very helpful in localization of tumors border [8]. The IOUS was able to well localize the lesions in $93 \%$ of their cases [8]. Other group studied the IOUS in pediatric brain tumors found that the IOUS was able to localize the lesions in all of their cases independent of the pathological diagnosis [4]. We had good localization of the lesions in $96 \%$ of our cases. In our results the IOUS differentiated solid from cystic parts in all patients with cystic component.

ii) Defining lesion borders:

Authors reported their experience with IOUS ability to define brain lesions borders. Their results showed that they were able to well define the borders in about $90 \%$ of their cases [8-10]. We were able to well define lesions in $87 \%$ of our cases. Authors correlate poor definition of IOUS to the lesions with low grade astrocytic tumors [4, 11-13]. In our study we had 2 cases astrocytoma grade III, which were fairly defined. We had only one case in our results, which was poorly defined by the IOUS. This case was a low-grade glioma (exophytic from the brainstem).

iii) Accuracy of the IOUS in determination of lesion volumes:

Le Roux et al concluded a strong correlation between the preoperative tumor volumes and margins of IOUS to that of computerized tomography scans in their study that included 22 patients and they concluded a strong correlation in their study group [14]. In the results of other group of researchers, they found the pre-resection IOUS imaging to be larger than the preoperative MRI imaging for gliomas and metastatic lesion [8].

In our study, which included 23 patients operated with the aid of the IOUS, there was no statistically significant difference between the volumes detected by the IOUS and that of preoperative MRI brain ( $p$ value 0.95). we also compared the volumes calculated from the IOUS for residual lesions after resection to that calculated from the postoperative MRI. We found statistically insignificant difference between the calculated lesion volumes ( $p$ value 0.94). This augments the results published by other authors regarding the ability of the IOUS to detect residual lesions in about $90 \%$ of their cases $[4,6]$.

iv) Extent of resection:

In a Cochrane review done by Barone et al for intraoperative neurosurgery imaging (Neuronavigation, iMRI, 5-aminolevuleinic acid and ultrasonography), they found low evidence in support of any of these imaging modalities to increase extent of resection of brain tumors. However, the ultrasonography or IOUS was under estimated or nonexistent in the recent literature surgery [15].

Intraoperative MRI and 5-ALA were reported by authors to be of value in improving the extent of resection, gross total resection was achieved in about $85 \%$ of cases with their assistance $[16,17]$. However, the 5-ALA is associated with a higher risk of postoperative morbidity [18]. Neuronavigation and Diffusion Tensor Imaging (DTI) associated systems were correlated with very low evidence in literature for their efficacy [15]. Still there is potential space for each institute to choose their compatible imaging guiding tool or combination [15]. Our results showed improvement of the extent of resection with the use of IOUS. We achieved gross total resection in 11/23 (49\%) cases.

v) Feasibility of the IOUS:

a) Real time images: Many studies have shown that, intraoperative brain shift is a source of errors in neurosurgical procedure with the neuronavigation [19-21].

Nimsky et al studied intraoperative brain shift. Their study was composed of a group of 64 patients who were operated for resection of different types of brain tumors. They used 0.2 Tesla open MRI for pre-resection and intraoperative imaging, and they found about $24 \mathrm{~mm}$ cortical displacement and about 
$4 \mathrm{~mm}$ displacement in deep margins of the tumors in $66 \%$ of cases. Their conclusion was supporting the concept of possible surgical errors with the dependence of neuronavigation systems without real-time updates [21]. It is reported that marked brain shift occurs with intraventricular or cystic lesions surgery due to loss of cerebrospinal fluid or cystic fluid [21]. Our results proved that the IOUS could provide real time intraoperative updates during resection of posterior fossa intra-axial lesions. In our study the IOUS was able to differentiate solid from cystic parts of the tumor in all the cases. Drainage of the cystic portion usually causes geometric distortion of the solid part in patients with cystic tumors [21].

b) Cost:

In a developing country like Egypt with a slowly growing economy; this was a big drive to start our study. It would not be practical to provide iMRI in all medical centers that serve the Egyptian population due to financial and space limitations. However, the ultrasound is already present in most of the hospitals and can be used in the brain tumor surgery with minimal cost. The mean direct additional costs of iMRI was found to be around 300 United states dollars per case [22]. However reports on the mean direct additional cost of the IOUS is around 30 USD per case $[4,11,12]$.

c) Additional time for surgery:

In our study the mean additional time of usage of the IOUS was 27 minutes in the whole surgery with a mean time for each scan of around 6.5 minutes. However, the mean additional time to surgery with the use of iMRI was found to be about 1.6 hours per surgery and about an hour for a single scan [23-26]. This would illustrate to what extent would the IOUS save time in comparison to the intraoperative MRI.

\subsection{Future Advances in IOUS}

Recently, the use of IOUS to update and correct the MRI images of the neuronavigation systems intraoperatively was reported [27]. By this way the IOUS would complete the missing part in the neuronavigation system, which is the real time update. This idea was first introduced by a group of researchers who were able to use a phantom to update the preoperative MRI images on the navigation system with an integration tool making real time updates available [27].

Their results were promising, and research continued. A group from Norway introduced "Sonowand" [28], which is a module capable to fuse the intraoperatively acquired 3D ultrasound images to the preoperative images on the Polaris navigation system. These fused images can be displayed either in split or composite images. They also quantified the degree of mismatch between the IOUS and MRI and they detected a degree of anatomical shift in $50 \%$ of their cases [28]. They published their 4 years' experience after that with detection of missed residual tumors in $53 \%$ of cases $[28,29]$.

Tirakotai et al published their novel IOUS platform with incorporation of the IOUS to the brain lab vector vision. Although this system allowed the fusion of IOUS images with the preoperative MRI and detection of degree of brain shift, none of them allowed the correction of this anatomical shift [30].

A group of researchers also published the contrastenhanced ultrasonography, in which they used the sulfur hexafluoride lipid filled microbubbles as a contrast agent which appear as hyper echoic in the IOUS. They found this technique very helpful in detection of the feeders of the lesions and the degree of vascularity in all of their cases [31].

\section{Conclusion}

Intraoperative ultrasound was found in our study to be of excellent value in resection of posterior fossa lesions with regards to: Modification of the craniectomy, localization of the lesion, detection of pre resection volume in comparison to preoperative MRI, definition of lesion borders, and differentiation of solid parts from cystic parts of the lesion. IOUS helps to detect residual volume accurately in comparison to the postoperative MRI. Mean additional time for the use of the IOUS was found to be 27 minutes in the whole surgery and 6.5 minutes for each scan. IOUS is a potential competitor (cost effective) to the intraoperative MRI in developing countries with limited resources like Egypt.

\section{Recommendations}

IOUS can be used in resection control of intra-axial posterior fossa lesion as a cost-effective device especially in developing countries. Intraoperative ultrasound is highly recommended in posterior fossa lesions with cystic components, it differentiates cystic from solid components and updates intraoperative surgical field changes after brain shift. Future researches and advances in the IOUS would include: neuronavigation platforms that are updated with IOUS images, intraoperative contrast enhanced ultrasonography, 5-ALA guided resection of brain lesions.

\section{Limitations}

IOUS is an operator dependent device that requires efficient training of the neurosurgeons to be able to interpret the IOUS images and correlate it to the MRI images. This problem can be resolved with the new neuro-navigation IOUS platforms, which fuse the IOUS images to the navigation systems.

\section{Declaration of Patient Consent}

The authors certify that they have obtained all appropriate patient consent forms. The patients understand that their names and initials will not be published.

\section{Conflicts of Interest}

There are no conflicts of interest. 


\section{References}

[1] Ius T, Isola M, Budai R, Pauletto G, Tomasino B, Fadiga L, et al. Low-grade glioma surgery in eloquent areas: volumetric analysis of extent of resection and its impact on overall survival. A single-institution experience in 190 patients. J Neurosurg. 2012; 117 (6): 1039-52.

[2] Maugeri R, Villa A, Pino M, Imperato A, Giammalva GR, Costantino G, et al. With a little help from my friends: The role of intraoperative fluorescent dyes in the surgical management of high-grade gliomas. Brain Sci. 2018; 8 (2): 31.

[3] Pino MA, Imperato A, Musca I, Maugeri R, Giammalva GR, Costantino G, et al. New hope in brain glioma surgery: The role of intraoperative ultrasound. A review. Brain Sci. 2018; 8 (11): 202

[4] El Beltagy MA, Aggag M, Kamal M. Role of intraoperative ultrasound in resection of pediatric brain tumors. Child's Nerv Syst. 2010; 26 (9): 1189-93.

[5] Karnofsky DA. The clinical evaluation of chemotherapeutic agents in cancer. Eval Chemother agents. 1949; 191-205.

[6] Ailon T, Dunham C, Carret A-S, Tabori U, Mcneely PD, Zelcer S, et al. The role of resection alone in select children with intracranial ependymoma: the Canadian Pediatric Brain Tumour Consortium experience. Child's Nerv Syst. 2015; 31 (1): 57-65.

[7] Zhang N, Ouyang T, Kang H, Long W, Thomas B, Zhu S. Adult medulloblastoma: clinical characters, prognostic factors, outcomes and patterns of relapse. J Neurooncol. 2015; 124 (2): 255-64.

[8] Hammoud MA, Ligon BL, Elsouki R, Shi WM, Schomer DF, Sawaya R. Use of intraoperative ultrasound for localizing tumors and determining the extent of resection: a comparative study with magnetic resonance imaging. J Neurosurg. 1996; 84 (5): 737-41

[9] Gerganov VM, Samii A, Akbarian A, Stieglitz L, Samii M, Fahlbusch R. Reliability of intraoperative high-resolution 2D ultrasound as an alternative to high-field strength MR imaging for tumor resection control: a prospective comparative study. J Neurosurg. 2009; 111 (3): 512-9.

[10] Tronnier VM, Bonsanto MM, Staubert A, Knauth M, Kunze S, Wirtz CR. Comparison of intraoperative MR imaging and 3Dnavigated ultrasonography in the detection and resection control of lesions. Neurosurg Focus. 2001; 10 (2): 1-5.

[11] Chacko AG, Kumar NKS, Chacko G, Athyal R, Rajshekhar V. Intraoperative ultrasound in determining the extent of resection of parenchymal brain tumours-a comparative study with computed tomography and histopathology. Acta Neurochir (Wien). 2003; 145 (9): 743-8.

[12] Wang $\mathrm{Y}$, Wang $\mathrm{Y}$, Wang $\mathrm{Y}$, Taniguchi $\mathrm{N}$, Chen X-C. Intraoperative real-time contrast-enhanced ultrasound angiography: a new adjunct in the surgical treatment of arteriovenous malformations. J Neurosurg. 2007; 107 (5): 959-64.

[13] Woydt M, Krone A, Soerensen N, Roosen K. Ultrasoundguided neuronavigation of deep-seated cavernous haemangiomas: clinical results and navigation techniques. $\mathrm{Br}$ J Neurosurg. 2001; 15 (6): 485-95.
[14] LeRoux PD, Berger MS, Ojemann GA, Wang K, Mack LA. Correlation of intraoperative ultrasound tumor volumes and margins with preoperative computerized tomography scans: An intraoperative method to enhance tumor resection. J Neurosurg. 1989; 71 (5): 691-8.

[15] Barone DG, Lawrie TA, Hart MG. Image guided surgery for the resection of brain tumours. Cochrane Database Syst Rev. 2014; (1).

[16] Roder C, Bisdas S, Ebner FH, Honegger J, Nägele T, Ernemann U, et al. Maximizing the extent of resection and survival benefit of patients in glioblastoma surgery: high-field iMRI versus conventional and 5-ALA-assisted surgery. Eur J Surg Oncol. 2014; 40 (3): 297-304.

[17] Schulder M, Carmel PW. Intraoperative magnetic resonance imaging: impact on brain tumor surgery. Cancer Control. 2003; 10 (2): 115-24.

[18] Puppa A Della, Ciccarino P, Lombardi G, Rolma G, Cecchin D, Rossetto M. 5-Aminolevulinic acid fluorescence in high grade glioma surgery: surgical outcome, intraoperative findings, and fluorescence patterns. Biomed Res Int. 2014; 2014.

[19] Hill DLG, Maurer CR, Maciunas RJ, Maciunas RJ, Barwise JA, Fitzpatrick JM, et al. Measurement of intraoperative brain surface deformation under a craniotomy. Neurosurgery. 1998; 43 (3): 514-26.

[20] Letteboer MMJ, Willems PWA, Viergever MA, Niessen WJ. Brain shift estimation in image-guided neurosurgery using 3D ultrasound. IEEE Trans Biomed Eng. 2005; 52 (2): 268-76.

[21] Nimsky C, Ganslandt O, Cerny S, Hastreiter P, Greiner G, Fahlbusch R. Quantification of, visualization of, and compensation for brain shift using intraoperative magnetic resonance imaging. Neurosurgery. 2000; 47 (5): 1070-80.

[22] Makary M, Chiocca EA, Erminy N, Antor M, Bergese SD, Abdel-Rasoul M, et al. Clinical and economic outcomes of low-field intraoperative MRI-guided tumor resection neurosurgery. J Magn Reson imaging. 2011; 34 (5): 1022-30.

[23] Samdani AF, Schulder M, Catrambone JE, Carmel PW. Use of a compact intraoperative low-field magnetic imager in pediatric neurosurgery. Child's Nerv Syst. 2005; 21 (2): 108-13.

[24] Schulder M. Intracranial surgery with a compact, low-fieldstrength magnetic resonance imager. Top Magn Reson Imaging. 2008; 19 (4): 179-89.

[25] Schulder M, Salas S, Brimacombe M, Fine P, Catrambone J, Maniker AH, et al. Cranial surgery with an expanded compact intraoperative magnetic resonance imager. J Neurosurg. 2006; 104 (4): 611-7.

[26] Schulder M, Azmi H, Biswal B. Functional magnetic resonance imaging in a low-field intraoperative scanner. Stereotact Funct Neurosurg. 2003; 80 (1-4): 125-31.

[27] Comeau RM, Sadikot AF, Fenster A, Peters TM. Intraoperative ultrasound for guidance and tissue shift correction in image-guided neurosurgery. Med Phys. 2000; 27 (4): 787-800.

[28] Lindseth F, Ommedal S, Bang J, Unsgård G, Hernes TAN. Image fusion of ultrasound and MRI as an aid for assessing anatomical shifts and improving overview and interpretation in ultrasound-guided neurosurgery. In: International Congress Series. Elsevier; 2001. p. 254-60. 
[29] Unsgaard G, Rygh OM, Selbekk T, Müller TB, Kolstad F, Lindseth $\mathrm{F}$, et al. Intra-operative $3 \mathrm{D}$ ultrasound in neurosurgery. Acta Neurochir (Wien). 2006; 148 (3): 235-53.

[30] Tirakotai W, Miller D, Heinze S, Benes L, Bertalanffy H, Sure U. A novel platform for image-guided ultrasound. Neurosurgery. 2006; 58 (4): 710-8.
[31] Prada F, Perin A, Martegani A, Aiani L, Solbiati L, Lamperti $\mathrm{M}$, et al. Intraoperative contrast-enhanced ultrasound for brain tumor surgery. Neurosurgery. 2014; 74 (5): 542-52. 